\title{
Concomitants of Dual Generalized Order Statistics from Morgenstern Type Bivariate Generalized Exponential Distribution
}

\author{
Saeid Tahmasebi* \\ Department of Statistics, Persian Gulf University, Bushehr, Iran \\ tahmasebi@pgu.ac.ir \\ Ali Akbar Jafari \\ Department of Statistics, Yazd University, Yazd, Iran \\ aajafari@yazd.ac.ir \\ Mahmoud Afshari \\ Department of Statistics, Persian Gulf University, Bushehr, Iran \\ afshar@pgu.ac.ir \\ Received 14 December 2012 \\ Accepted 6 October 2014
}

\begin{abstract}
[9] introduced the concept of dual generalized order statistics (dgos) that enables a common approach to descending ordered random variables like reversed order statistics and lower record values. In this paper, we have obtained probability density function (pdf) of $r$-th, and the joint pdf of $r$-th and $s$-th, concomitants of dgos from Morgenstern type bivariate generalized exponential distribution and derived their product moments. Further the results are deduced for moments of $k$-th lower record values and order statistics. Recurrence relations between moments of concomitants are also obtained. Finally, some properties of joint distributions for concomitants of dgos are presented.
\end{abstract}

Keywords: Concomitant; Dual generalized order statistics; Generalized exponential distribution; Record values.

2000 Mathematics Subject Classification: 62E15, 62G30

\section{Introduction}

[12] introduced the concept of generalized order statistics (gos) as a unified models of ordered random variables such as ordinary order statistics, sequential order statistics, progressive type II censoring, record values and Pfeifers records. The concept of lower generalized order statistics was given by [19], and later [9] introduced it as dual generalized order statistics (dgos) to enable a common approach to descendingly ordered random variables like reversed order statistics and lower records models. There is a connection between the concepts of gos and dgos.

Let $X$ be an absolutely continuous random variables with cumulative distribution function (cdf) $F$ and the probability density function (pdf) $f$. For $n \in \mathbb{N}, k \geq 1, \tilde{m}=\left(m_{1}, m_{2}, \ldots, m_{n-1}\right) \in \mathbb{R}^{n-1}$, the

${ }^{*}$ Corresponding 
random variables $X_{d}=\left(X_{d}(1, n, \tilde{m}, k), X_{d}(2, n, \tilde{m}, k), \ldots, X_{d}(n, n, \tilde{m}, k)\right)$ are called dgos if their joint pdf is given by

$$
f_{X_{d}}\left(x_{1}, x_{2}, \ldots, x_{n}\right)=k C_{n-1}\left(\prod_{i=1}^{n-1}\left[F\left(x_{i}\right)\right]^{m_{i}} f\left(x_{i}\right)\right)\left[F\left(x_{n}\right)\right]^{k-1} f\left(x_{n}\right),
$$

on the cone $\left\{\left(x_{1}, \ldots, x_{n}\right): F^{-1}(1)>x_{1} \geq x_{2} \geq \ldots \geq x_{n}>F^{-1}(0)\right\} \subset \mathbb{R}^{n}$, such that $C_{r}=\prod_{j=1}^{r} \gamma_{j}$ and $\gamma_{j}=k+n-j+\sum_{h=j}^{n-1} m_{h} \geq 1$ for all $j \in\{1,2, \ldots, n\}$.

An important special case in the concept of dgos is choosing $m$ according to $m_{i}=m$. With taking $m=0$ and $k=1$, the random variable $X_{d}(r, n, m, k)$ reduces to the $(n-r+1)$-th order statistics, and with taking $m=-1$, the random variable $X_{d}(r, n, m, k)$ reduces to $r$-th, $k$-lower record value. For more details and some applications of dgos or lower generalized order statistics, reader can refer to $[1,2,4,5,7,9,13-16,19]$.

The marginal pdf of $r$ th dgos, $X_{d}(r, n, m, k)$ is

$$
f_{X_{d}(r, n, m, k)}(x)=\frac{C_{r-1}}{(r-1) !}[F(x)]^{\gamma_{r}-1} f(x) g_{m}^{r-1}(F(x)),
$$

and the joint pdf of $r$ th and sth dgos's, $X_{d}(r, s)=\left(X_{d}(r, n, m, k), X_{d}(s, n, m, k)\right), 1 \leq r<s \leq n$, is

$$
\begin{aligned}
f_{X_{d}(r, s)}(x, y)= & \frac{C_{s-1}}{(r-1) !(s-r-1) !}[F(x)]^{m} f(x) g_{m}^{r-1}(F(x)) \\
& \times\left[h_{m}(F(y))-h_{m}(F(x))\right]^{s-r-1}[F(x)]^{\gamma_{s}-1} f(y), \quad x<y,
\end{aligned}
$$

where $g_{m}(t)=h_{m}(t)-h_{1}(t), t \in(0,1)$ and

$$
h_{m}(t)= \begin{cases}-(m+1)^{-1} t^{m+1} & m \neq-1, \\ -\log (t) & m=-1 .\end{cases}
$$

Let $\left(X_{1}, Y_{1}\right),\left(X_{2}, Y_{2}\right), \ldots,\left(X_{n}, Y_{n}\right)$ be $n$ pairs of independent random variables from a bivariate population with cdf $F(x, y)$. If the $X$-variates are arranged in descending order as $X_{d}(1, n, m, k) \geq$ $X_{d}(2, n, m, k) \geq \ldots \geq X_{d}(n, n, m, k)$, then $Y$-variates paired (not necessarily in descending order) with these dgos are called the concomitants of dgos and denoted by $Y_{[1, n, m, k]}, Y_{[2, n, m, k]}, \ldots, Y_{[n, n, m, k]}$. The pdf of $Y_{[r, n, m, k]}$, the $r$-th concomitant dgos, is given as ( [18])

$$
g_{[r, n, m, k]}(y)=\int_{-\infty}^{+\infty} f_{Y \mid X}(y \mid x) f_{X_{d}(r, n, m, k)}(x) d x
$$

and the joint pdf of $Y_{[r, n, m, k]}$ and $Y_{[r, n, m, k]} 1 \leq r<s \leq n$ is given as

$$
g_{[r, s, n, m, k]}(y)=\int_{-\infty}^{+\infty} \int_{-\infty}^{x_{1}} f_{Y \mid X}\left(y_{1} \mid x_{1}\right) f_{Y \mid X}\left(y_{2} \mid x_{2}\right) f_{X_{d}(r, s)}\left(x_{1}, x_{2}\right) d x_{1} d x_{2}, \quad x_{1}<x_{2} .
$$

Recently, [20] defined the Morgenstern type bivariate generalized exponential distribution (MTBGED). This distribution is a special case of Morgenstern family, defined by [17] with considering the generalized exponential (GE) distribution ( [11]) as the marginal distribution function. In this paper, we study and derive the properties of concomitants of dgos in MTBGED. Similar 
works are done for dgos and gos in some literature: [18] considered the concomitants of dgos for Morgenstern type bivariate power function distribution.

$[3,6,8,20]$ studied the concomitants of gos for Morgenstern type bivariate exponential distribution (MTBED), Gumbel's bivariate exponential distribution, Morgenstern family, and MTBGED, respectively.

This paper is organized as follows: in Section 2, we will review the MTBGED and some of its properties. In Section 3, we study the properties of the marginal distributions of concomitants for dgos from MTBGED. The properties of joint distributions of these statistics in Section 4.

\section{A review on MTBGED}

[11] defined the GE distribution with the following cdf

$$
F_{X}(x)=\left(1-e^{-\theta x}\right)^{\alpha}, \quad x>0, \theta>0, \alpha>0 .
$$

and we denote this cdf by $G E(\theta, \alpha)$.

By using the binomial series expansion, the $k$ th moment of a random variable with $G E(\theta, \alpha)$ is given as

$$
\mu_{k}=\frac{\alpha \Gamma(k+1)}{\theta^{k}} \sum_{i=0}^{\infty} \frac{(-1)^{i}}{(i+1)^{k+1}} A(\alpha-1, i),
$$

where $A(\alpha-1, i)=\left(\begin{array}{c}\alpha-1 \\ i\end{array}\right)$. Also, its moment generating function is $M_{X}(t)=\alpha \operatorname{Beta}(\alpha, 1-t / \theta)$ where $\operatorname{Beta}(a, b)=\frac{\Gamma(a) \Gamma(b)}{\Gamma(a+b)}$.

With considering the GE distribution, [20] defined the MTBGED as a special case of Morgenstern family. The random variables $X$ and $Y$ have MTBGED if their joint cdf is

$$
\begin{array}{r}
F_{X, Y}(x, y)=\left(1-e^{-\theta_{1} x}\right)^{\alpha_{1}}\left(1-e^{-\theta_{2} y}\right)^{\alpha_{2}}\left\{1+\lambda\left[1-\left(1-e^{-\theta_{1} x}\right)^{\alpha_{1}}\right]\left[1-\left(1-e^{-\theta_{2} y}\right)^{\alpha_{2}}\right]\right\}, \\
x, y>0,-1 \leq \lambda \leq 1 .
\end{array}
$$

The corresponding pdf of this distribution is given as

$$
\begin{aligned}
f_{X, Y}(x, y)= & \alpha_{1} \alpha_{2} \theta_{1} \theta_{2} e^{-\theta_{1} x-\theta_{2} y}\left(1-e^{-\theta_{1} x}\right)^{\alpha_{1}-1}\left(1-e^{-\theta_{2} y}\right)^{\alpha_{2}-1} \\
& \times\left\{1+\lambda\left[2\left(1-e^{-\theta_{1} x}\right)^{\alpha_{1}}-1\right]\left[2\left(1-e^{-\theta_{2} y}\right)^{\alpha_{2}}-1\right]\right\} .
\end{aligned}
$$

Note that this distribution is a extension of MTBED introduced by [10].

The moments of the MTBGED are given as

$$
E\left(X^{n} Y^{m}\right)=E\left(X^{n}\right) E\left(Y^{m}\right)+\lambda\left(E\left(U^{n}\right)-E\left(X^{n}\right)\right)\left(E\left(V^{m}\right)-E\left(Y^{m}\right)\right),
$$

where $U$ and $V$ are independent random variables with $G E\left(\theta_{1}, 2 \alpha_{1}\right)$ and $G E\left(\theta_{2}, 2 \alpha_{2}\right)$, respectively. Also,

$$
\begin{array}{ll}
\mu_{x}=E(X)=\frac{B\left(\alpha_{1}\right)}{\theta_{1}}, & \mu_{y}=E(Y)=\frac{B\left(\alpha_{2}\right)}{\theta_{2}}, \\
\sigma_{x}^{2}=\operatorname{Var}(X)=\frac{C\left(\alpha_{1}\right)}{\theta_{1}^{2}}, & \sigma_{y}^{2}=\operatorname{Var}(Y)=\frac{C\left(\alpha_{2}\right)}{\theta_{2}^{2}},
\end{array}
$$


Tahmasebi, Jafari, Afshari

$$
\begin{aligned}
& \mu_{x y}=E(X Y)=\frac{B\left(\alpha_{1}\right) B\left(\alpha_{2}\right)+\lambda D\left(\alpha_{1}\right) D\left(\alpha_{2}\right)}{\theta_{1} \theta_{2}}, \\
& \rho_{x y}=\operatorname{Corr}(X, Y)=\frac{\lambda D\left(\alpha_{1}\right) D\left(\alpha_{2}\right)}{\sqrt{C\left(\alpha_{1}\right) C\left(\alpha_{2}\right)}},
\end{aligned}
$$

where $B(\alpha)=\psi(\alpha+1)-\psi(1), C(\alpha)=\psi^{\prime}(1)-\psi^{\prime}(\alpha+1)$, and $D\left(\alpha_{1}\right)=B\left(2 \alpha_{1}\right)-B\left(\alpha_{1}\right)$, and $\psi($.$) is the digamma function and \psi^{\prime}($.$) is its derivative.$

The conditional distribution of $Y$ given $X=x$ has the pdf

$$
f_{Y \mid X}(y \mid x)=f_{Y}(y)\left[1+\lambda\left(2 F_{Y}(y)-1\right)\left(2 F_{X}(x)-1\right)\right],
$$

Therefore, the regression curve of $Y$ given $X=x$ for MTBGED is

$$
\begin{aligned}
E(Y \mid X=x) & =E(Y)+\lambda\left(2 F_{X}(x)-1\right)(E(V)-E(Y)) \\
& =\mu_{y}\left[1+\frac{\lambda D\left(\alpha_{2}\right)}{B\left(\alpha_{2}\right)}\left(2\left(1-e^{-\theta_{1} x}\right)^{\alpha_{1}}-1\right)\right],
\end{aligned}
$$

where $V$ has $G E\left(\theta_{2}, 2 \alpha_{2}\right)$.

\section{Concomitants of dgos in MTBGED}

In this section, we consider the concomitants of dgos in MTBGED and obtain the properties of their marginal distributions. Also, some recurrence relations between moments of concomitants are presented.

\subsection{Marginal distribution of concomitants}

Consider $X$ and $Y$ are random variables from a Morgenstern family with marginal cdf $F_{X}(x)$ and $F_{Y}(y)$, and marginal pdf $f_{X}(x)$ and $f_{Y}(y)$, respectively.

Also, let $X_{d}(1, n, m, k), X_{d}(2, n, m, k), \ldots, X_{d}(n, n, m, k)$ be dgos for a random sample this family, and $Y_{[1, n, m, k]}, Y_{[2, n, m, k]}, \ldots, Y_{[n, n, m, k]}$ be the concomitants of corresponding to this dgos.

[18] using (1.4) show that the marginal distribution of $Y_{[r, n, m, k]}$ is

$$
\begin{aligned}
g_{[r, n, m, k]}(y) & =f_{Y}(y)\left[1+\lambda C_{r, n}^{*}\left(1-2 F_{Y}(y)\right)\right] \\
& =f_{1: 1}(y)+\lambda C_{r, n}^{*}\left[f_{1: 1}(y)-f_{2: 2}(y)\right],
\end{aligned}
$$

where $C_{r, n}^{*}=1-\frac{2 \prod_{j=1}^{r} \gamma_{j}}{\prod_{j=1}^{r}\left(\gamma_{j}+1\right)}$ and $f_{i: n}(y)$ is the pdf of $Y_{i, n}$, the $i$ th order statistic of a random sample of size $n$ of $Y$. Note that $g_{[r, n, m, k]}(y)$ depends only on the marginal distribution of $Y$ and the distribution of $Y_{2,2}$ ( [8]).

Using (3.1), the pdf of $Y_{[r, n, m, k]}$ for MTBGED is obtained as

$$
g_{[r, n, m, k]}(y)=\alpha_{2} \theta_{2} e^{-\theta_{2} y}\left(1-e^{-\theta_{2} y}\right)^{\alpha_{2}-1}\left[1+\delta_{r}-2 \delta_{r}\left(1-e^{-\theta_{2} y}\right)^{\alpha_{2}}\right],
$$

where $\delta_{r}=C_{r}^{*} \lambda$. Obviously, we can find that

$$
g_{[r, n, m, k]}(y)=\left(1+\delta_{r}\right) f_{1: 1}(y)-\delta_{r} f_{2: 2}(y)=\left(1+\delta_{r}\right) f_{Y}(y)-\delta_{r} f_{V}(y),
$$


where $f_{Y}(y)$ and $f_{V}(y)$ are pdf's of random variables $Y$ and $V$ with $G E\left(\theta_{2}, \alpha_{2}\right)$ and $G E\left(\theta_{2}, 2 \alpha_{2}\right)$, respectively.

Remark 3.1. With taking $m=0$ and $k=1$ in (3.2), the pdf of $(n-r+1)$-th concomitant of order statistic from MTBGED is given as

$$
\begin{array}{r}
g_{[n-r+1: n]}(y)=\alpha_{2} \theta_{2} e^{-\theta_{2} y}\left(1-e^{-\theta_{2} y}\right)^{\alpha_{2}-1}\left[1+\lambda\left\{1-\frac{2(n-r+1)}{n+1}\right\}\left(1-2\left(1-e^{-\theta_{2} y}\right)^{\alpha_{2}}\right)\right], \\
1 \leq r \leq n .
\end{array}
$$

Note that $\frac{1}{n} \sum_{r=1}^{n} g_{[n-r+1: n]}(y)=f_{Y}(y)$.

Remark 3.2. With taking $m=-1$ in (3.2), the pdf of $r$-th concomitant of $k$-lower record value from MTBGED is given as

$$
g_{[r, n,-1, k]}(y)=\alpha_{2} \theta_{2} e^{-\theta_{2} y}\left(1-e^{-\theta_{2} y}\right)^{\alpha_{2}-1}\left[1+\lambda\left\{1-2\left(\frac{1+2 k}{k+1}\right)^{r}\right\}\left(1-2\left(1-e^{-\theta_{2} y}\right)^{\alpha_{2}}\right)\right],
$$

\subsection{Moment generating function and moments of $Y_{[r, n, m, k]}$}

Using (3.3), the moment generating function(mgf) of $Y_{[r, n, m, k]}$ is given as

$$
\begin{aligned}
M_{[r, n, m, k]}(t) & =\left(1+\delta_{r}\right) M_{Y}(t)-\delta_{r} M_{V}(t) \\
& =\alpha_{2}\left[\left(1+\delta_{r}\right) \operatorname{Beta}\left(\alpha_{2}, 1-\frac{t}{\theta_{2}}\right)-2 \delta_{r} \operatorname{Beta}\left(2 \alpha_{2}, 1-\frac{t}{\theta_{2}}\right)\right],
\end{aligned}
$$

where $M_{Y}(t)$ and $M_{V}(t)$ are moment generating functions of random variables $Y$ and $V$, respectively. With differentiating (3.6) with respect to $t$ and using (2.2), we get the $l$ th moment of $Y_{[r, n, m, k]}$ as

$$
\begin{aligned}
\mu_{[r, n, m, k]}^{(l)} & =E\left(Y_{[r, n, m, k]}^{l}\right)=\left(1+\delta_{r}\right) E\left(Y^{l}\right)-\delta_{r} E\left(V^{l}\right) \\
& =\sum_{i=0}^{\infty}(-1)^{i} \frac{\alpha_{2} \Gamma(l+1)}{\theta_{2}^{l}(i+1)^{l+1}}\left[\left(1+\delta_{r}\right) A\left(\alpha_{2}-1, i\right)-2 \delta_{r} A\left(2 \alpha_{2}-1, i\right)\right] .
\end{aligned}
$$

Since (3.7) is a convergent series for any $l \geq 0$, so all the moments exist for integer values of $\alpha_{2}$. With putting $l=1$, we obtain the mean as

$$
\begin{aligned}
\mu_{[r, n, m, k]} & =E\left(Y_{[r, n, m, k]}\right)=\frac{1}{\theta_{2}}\left[B\left(\alpha_{2}\right)-\delta_{r} D\left(\alpha_{2}\right)\right] \\
& =\sum_{i=0}^{\infty}(-1)^{i} \frac{\alpha_{2}}{\theta_{2}(i+1)^{2}}\left[\left(1+\delta_{r}\right) A\left(\alpha_{2}-1, i\right)-2 \delta_{r} A\left(2 \alpha_{2}-1, i\right)\right] .
\end{aligned}
$$

In general, if $h(y)$ is a measurable function of $y$, then

$$
E\left(h\left(Y_{[r, n, m, k]}\right)\right)=\left(1+\delta_{r}\right) E(h(Y))-\delta_{r} E(h(V)),
$$

and

$$
\begin{aligned}
E\left\{h\left(Y_{[r, n, m, k]}\right)\right\}-E\left\{h\left(Y_{[r-1, n, m, k]}\right)\right\} & =\left[\delta_{r}-\delta_{r-1}\right][E(h(Y))-E(h(V))] \\
& =2 \lambda\left[\prod_{j=1}^{r-1} \frac{\gamma_{j}}{\gamma_{j}+1}-\prod_{j=1}^{r-1} \frac{\gamma_{j+1}}{\gamma_{j+1}+1}\right][E(h(Y))-E(h(V))]
\end{aligned}
$$


Tahmasebi, Jafari, Afshari

$$
=2 \lambda\left[\frac{\gamma_{1} \gamma_{2} \ldots \gamma_{r-1}}{\left(\gamma_{1}+1\right)\left(\gamma_{2}+1\right) \ldots\left(\gamma_{r-1}+1\right)\left(\gamma_{r}+1\right)}\right][E(h(Y))-E(h(V))]
$$

Remark 3.3. With taking $m=0$ and $k=1$ in (3.8), the mean of $(n-r+1)$-th concomitant of order statistic from MTBGED is given as

$$
\mu_{[n-r+1: n]}=\frac{1}{\theta_{2}}\left[B\left(\alpha_{2}\right)-\lambda\left\{1-\frac{2(n-r+1)}{n+1}\right\} D\left(\alpha_{2}\right)\right] .
$$

Therefore, the following recurrence relations between the means of concomitants of order statistics similar to [20] are obtained

$$
\begin{aligned}
& \mu_{[r+1: n]}=\frac{\mu_{[r+2: n]}+\mu_{[r: n]}}{2}=\mu_{[r: n]}+\frac{2 \lambda D\left(\alpha_{2}\right)}{(n+1) \theta_{2}}, \\
& \mu_{[r: n]}-\mu_{[r: n-1]}=\frac{-\lambda D\left(\alpha_{2}\right)}{(n+1) \theta_{2}}, \\
& \mu_{[r: n]}-\mu_{[r-i: n]}=\frac{2 \lambda i D\left(\alpha_{2}\right)}{(n+1) \theta_{2}}, \quad 1 \leq i \leq r-1, \\
& \mu_{[r: n]}-\mu_{[r: n-j]}=\frac{-\lambda j D\left(\alpha_{2}\right)}{(n+1) \theta_{2}}, \quad 1 \leq j \leq n-r, \\
& \mu=\frac{1}{n} \sum_{r=1}^{n} \mu_{[n-r+1: n]}=\frac{B\left(\alpha_{2}\right)}{\theta_{2}}, \\
& \mu_{[r: n]}=\mu_{[1: n]}+\frac{2 \lambda(r-1) D\left(\alpha_{2}\right)}{(n+1) \theta_{2}}, \quad 1 \leq r \leq n, \\
& \mu_{[r: n]}=\mu_{[r: r]}-\frac{\lambda(n-r) D\left(\alpha_{2}\right)}{(n+1) \theta_{2}}, \quad 1 \leq r \leq n, \\
& \mu_{[n-r+1: n]}=\mu_{[r: n]}+\frac{2 \lambda(n-2 r+1) D\left(\alpha_{2}\right)}{(n+1) \theta_{2}}, \\
& \mu_{[r: n]}=\mu_{[r \beta:(n+1) \beta-1]}, \quad \beta \geq 1, \\
& \mu_{[r: n]}=\sum_{s=n-r+1}^{n}(-1)^{s-n+r-1}\left(\begin{array}{l}
s-1 \\
n-r
\end{array}\right)\left(\begin{array}{l}
n \\
s
\end{array}\right) \mu_{[1: s]} .
\end{aligned}
$$

Remark 3.4. Set $m=-1$ in (3.8), to get the mean of $r$-th concomitant of $k$-lower record value from MTBGED as

$$
\mu_{[r, n,-1, k]}=\frac{1}{\theta_{2}}\left[B\left(\alpha_{2}\right)-\lambda\left\{1-2\left(\frac{1+2 k}{k+1}\right)^{r}\right\} D\left(\alpha_{2}\right)\right] .
$$

An explicit expression of Shannon entropy for concomitants of dgos in Morgenstern family is given as

$$
H\left(Y_{[r, n, m, k]}\right)=E\left[-\log g_{[r, n, m, k]}(Y)\right]=Z_{r, n, m, k}+H(Y)\left(1+\delta_{r}\right)+2 \delta_{r} \phi_{f}(u),
$$

where

$$
Z_{r, n, m, k}=\frac{1}{4 \delta_{r}}\left\{\left(1+\delta_{r}\right)^{2} \log \left(1+\delta_{r}\right)-\left(1-\delta_{r}\right)^{2} \log \left(1-\delta_{r}\right)\right\}+\frac{1}{2}
$$


and $\phi_{f}(u)=\int_{0}^{1} u \log f_{Y}\left(F_{Y}^{-1}(u)\right) d u$. Applying this expression for $Y_{[r: n]}$ in MTBGED, we have ( [20])

$$
H\left(Y_{[r: n]}\right)=W_{\lambda, n}(r)-\log \left(\alpha_{2} \theta_{2}\right)+B\left(\alpha_{2}\right)-\lambda\left(\frac{n-2 r+1}{n+1}\right) D\left(\alpha_{2}\right)+\frac{\alpha_{2}-1}{\alpha_{2}}\left[1+\frac{\lambda\left(\frac{n-2 r+1}{n+1}\right)}{2}\right],
$$

where

$$
\begin{aligned}
W_{\lambda, n}(r) & =\frac{1}{8 \lambda\left(\frac{n-2 r+1}{n+1}\right)}\left\{\left(1-\lambda\left(\frac{n-2 r+1}{n+1}\right)\right)^{2}\left[2 \log \left(1-\lambda\left(\frac{n-2 r+1}{n+1}\right)\right)-1\right]\right. \\
& \left.-\left(1+\lambda\left(\frac{n-2 r+1}{n+1}\right)\right)^{2}\left[2 \log \left(1+\lambda\left(\frac{n-2 r+1}{n+1}\right)\right)-1\right]\right\}
\end{aligned}
$$

\subsection{Some Recurrence relations}

In this section we shall present several recurrence relations between pdf's, moments and mgf's of concomitants. From (3.3), we have

$$
\begin{aligned}
& g_{[r, n, m, k]}(y)=f_{1: 1}(y)+C^{*}(r, n, m, k) \lambda\left[f_{1: 1}(y)-f_{2: 2}(y)\right], \\
& g_{[r, n, m, k]}(y)-g_{[r-1, n, m, k]}(y)=2 \lambda\left[\prod_{j=1}^{r} \frac{\gamma_{j}}{\gamma_{j}+1}-\prod_{j=1}^{r-1} \frac{\gamma_{j}}{\gamma_{j}+1}\right]\left[f_{2: 2}(y)-f_{1: 1}(y)\right], \\
& g_{[r, n, m, k]}(y)-g_{[r-1, n-1, m, k]}(y)=2 \lambda\left[\prod_{j=1}^{r} \frac{\gamma_{j}}{\gamma_{j}+1}-\prod_{j=1}^{r-1} \frac{\gamma_{j+1}}{\gamma_{j+1}+1}\right]\left[f_{2: 2}(y)-f_{1: 1}(y)\right], \\
& g_{[r-1, n, m, k]}(y)-g_{[r-1, n-1, m, k]}(y)=2 \lambda\left[\prod_{j=1}^{r-1} \frac{\gamma_{j}}{\gamma_{j}+1}-\prod_{j=1}^{r-1} \frac{\gamma_{j+1}}{\gamma_{j+1}+1}\right]\left[f_{2: 2}(y)-f_{1: 1}(y)\right] .
\end{aligned}
$$

Also, for $1 \leq i \leq n-r$ and $1 \leq j \leq r-1$, we have

$$
\begin{aligned}
& g_{[r, n, m, k]}(y)-g_{[r, n-i, m, k]}(y)=\lambda\left[C^{*}(r, n, m, k)-C^{*}(r, n-i, m, k)\right]\left[f_{1: 1}(y)-f_{2: 2}(y)\right], \\
& g_{[r, n, m, k]}(y)-g_{[r-j, n, m, k]}(y)=\lambda\left[C^{*}(r, n, m, k)-C^{*}(r-j, n, m, k)\right]\left[f_{1: 1}(y)-f_{2: 2}(y)\right], \\
& g_{[r, n, m, k]}(y)-g_{[r-j, n-i, m, k]}(y)=\lambda\left[C^{*}(r, n, m, k)-C^{*}(r-j, n-i, m, k)\right]\left[f_{1: 1}(y)-f_{2: 2}(y)\right] .
\end{aligned}
$$

Using (3.7) the following recurrence relations between moments of concomitants are valid:

$$
\begin{aligned}
& \mu_{[r, n, m, k]}^{(l)}-\mu_{[r, n-i, m, k]}^{(l)}=\lambda\left[C^{*}(r, n, m, k)-C^{*}(r, n-i, m, k)\right]\left[E\left(Y^{l}\right)-E\left(V^{l}\right)\right], \\
& \mu_{[r, n, m, k]}^{(l)}-\mu_{[r-j, n, m, k]}^{(l)}=\lambda\left[C^{*}(r, n, m, k)-C^{*}(r-j, n, m, k)\right]\left[E\left(Y^{l}\right)-E\left(V^{l}\right)\right], \\
& \mu_{[r, n, m, k]}^{(l)}-\mu_{[r-j, n-i, m, k]}^{(l)}=\lambda\left[C^{*}(r, n, m, k)-C^{*}(r-j, n-i, m, k)\right]\left[E\left(Y^{l}\right)-E\left(V^{l}\right)\right],
\end{aligned}
$$

where $E\left(Y^{l}\right)=\mu_{1: 1}^{l}$ and $E\left(V^{l}\right)=\mu_{2: 2}^{l}$. Furthermore, for $i, j=1$, we have

$$
\begin{aligned}
& \mu_{[r, n, m, k]}^{(l)}-\mu_{[r, n-1, m, k]}^{(l)}=2 r \lambda(m+1)\left[\frac{\gamma_{2} \gamma_{3} \ldots \gamma_{r}}{\left(\gamma_{1}+1\right)\left(\gamma_{2}+1\right) \ldots\left(\gamma_{r}+1\right)\left(\gamma_{r+1}+1\right)}\right]\left[\mu_{2: 2}^{l}-\mu_{1: 1}^{l}\right], \\
& \mu_{[r, n, m, k]}^{(l)}-\mu_{[r-1, n, m, k]}^{(l)}=2 \lambda\left[\frac{\gamma_{1} \gamma_{2} \ldots \gamma_{r-1}}{\left(\gamma_{1}+1\right)\left(\gamma_{2}+1\right) \ldots\left(\gamma_{r-1}+1\right)\left(\gamma_{r}+1\right)}\right]\left[\mu_{1: 1}^{l}-\mu_{2: 2}^{l}\right] .
\end{aligned}
$$

For $1 \leq i_{1} \leq i_{2} \leq n-r$ and $1 \leq j_{1} \leq j_{2} \leq r-1$, the relation between mgf's of concomitants are

$$
M_{[r, n, m, k]}(t)-M_{\left[r, n-i_{1}, m, k\right]}(t)=\alpha_{2} \lambda\left[C^{*}(r, n, m, k)-C^{*}\left(r, n-i_{1}, m, k\right)\right]\left[\operatorname{Beta}\left(\alpha_{2}, 1-\frac{t}{\theta_{2}}\right)\right.
$$




$$
\begin{gathered}
\left.-2 \operatorname{Beta}\left(2 \alpha_{2}, 1-\frac{t}{\theta_{2}}\right)\right] \\
M_{[r, n, m, k]}(t)-M_{\left[r-j_{1}, n, m, k\right]}(t)=\alpha_{2} \lambda\left[C^{*}(r, n, m, k)-C^{*}\left(r-j_{1}, n, m, k\right)\right]\left[\operatorname{Beta}\left(\alpha_{2}, 1-\frac{t}{\theta_{2}}\right)\right. \\
\left.-2 \operatorname{Beta}\left(2 \alpha_{2}, 1-\frac{t}{\theta_{2}}\right)\right], \\
M_{[r, n, m, k]}(t)-M_{\left[r-j_{1}, n-i_{1}, m, k\right]}(t)=\alpha_{2} \lambda\left[C^{*}(r, n, m, k)-C^{*}\left(r-j_{1}, n-i_{1}, m, k\right)\right]\left[\operatorname{Beta}\left(\alpha_{2}, 1-\frac{t}{\theta_{2}}\right)\right. \\
\left.-2 \operatorname{Beta}\left(2 \alpha_{2}, 1-\frac{t}{\theta_{2}}\right)\right], \\
M_{\left[r-j_{1}, n-i_{1}, m, k\right]}(t)-M_{\left[r-j_{2}, n-i_{2}, m, k\right]}(t)=\alpha_{2} \lambda\left(C^{*}\left(r-j_{1}, n-i_{1}, m, k\right)-C^{*}\left(r-j_{2}, n-i_{2}, m, k\right)\right) \\
\times\left[\operatorname{Beta}\left(\alpha_{2}, 1-\frac{t}{\theta_{2}}\right)-2 \operatorname{Beta}\left(2 \alpha_{2}, 1-\frac{t}{\theta_{2}}\right)\right] .
\end{gathered}
$$

If we take $m=0$ and $k=1$, then the $l$ th moment and $\operatorname{mgf}$ of $Y_{[r: n]}$ can be deduced from (3.6) and (3.8), respectively as

$$
\begin{aligned}
E\left[Y_{[r: n]}^{l}\right]= & {\left[1+\lambda\left(\frac{n-2 r+1}{n+1}\right)\right] \mu_{1: 1}^{l}-\lambda\left(\frac{n-2 r+1}{n+1}\right) \mu_{2: 2}^{l} } \\
= & \sum_{i=0}^{\infty}(-1)^{i} \frac{\alpha_{2} \Gamma(l+1)}{\theta_{2}^{l}(i+1)^{l+1}}\left[\left(1+\lambda\left(\frac{n-2 r+1}{n+1}\right)\right) A\left(\alpha_{2}-1, i\right)\right. \\
& \left.-2 \lambda\left(\frac{n-2 r+1}{n+1}\right) A\left(2 \alpha_{2}-1, i\right)\right], \\
M_{[r: n]}(t)= & \alpha_{2}\left[1+\lambda\left(\frac{n-2 r+1}{n+1}\right)\right] \operatorname{Beta}\left(\alpha_{2}, 1-\frac{t}{\theta_{2}}\right)-2 \lambda \alpha_{2}\left(\frac{n-2 r+1}{n+1}\right) \operatorname{Beta}\left(2 \alpha_{2}, 1-\frac{t}{\theta_{2}}\right) .
\end{aligned}
$$

If we take $m=-1$, then the $l$ th moment and mgf of $r$-th concomitant of k-lower record value can be deduced from (3.6) and (3.8), respectively as

$$
\begin{aligned}
\mu_{[r, n,-1, k]}^{(l)}= & {\left[1+\lambda\left\{1-2\left(\frac{1+2 k}{k+1}\right)^{r}\right\}\right] \mu_{1: 1}^{l}+\lambda\left\{1-2\left(\frac{1+2 k}{k+1}\right)^{r}\right\} \mu_{2: 2}^{l} } \\
= & \sum_{i=0}^{\infty}(-1)^{i} \frac{\alpha_{2} \Gamma(l+1)}{\theta_{2}^{l}(i+1)^{l+1}}\left[\left(1+\lambda\left\{1-2\left(\frac{1+2 k}{k+1}\right)^{r}\right\}\right) A\left(\alpha_{2}-1, i\right)\right. \\
& \left.+2 \lambda\left\{1-2\left(\frac{1+2 k}{k+1}\right)^{r}\right\} A\left(2 \alpha_{2}-1, i\right)\right], \\
M_{[r, n,-1, k]}(t)= & \alpha_{2}\left[1+\lambda\left\{1-2\left(\frac{1+2 k}{k+1}\right)^{r}\right\}\right] \operatorname{Beta}\left(\alpha_{2}, 1-\frac{t}{\theta_{2}}\right) \\
& -2 \alpha_{2} \lambda\left\{1-2\left(\frac{1+2 k}{k+1}\right)^{r}\right\} \operatorname{Beta}\left(2 \alpha_{2}, 1-\frac{t}{\theta_{2}}\right) .
\end{aligned}
$$

\section{Joint distribution of two concomitants}

In this Section, we obtain the joint distribution of concomitants of two dgos, and study their properties. 
Let $Y_{[r, n, m, k]}$ and $Y_{[s, n, m, k]}, 1 \leq r<s \leq n$ be concomitants of the $r$-th and $s$-th dgos from a Morgenstern family. The joint pdf of $Y_{[r, n, m, k]}$ and $Y_{[r, n, m, k]}$ is ( [18])

$$
\begin{aligned}
g_{[r, s, n, m, k]}(y)= & f_{Y}\left(y_{1}\right) f_{Y}\left(y_{2}\right)\left[1+\lambda\left(1-2 F_{Y}\left(y_{1}\right)\right)\left[1-2 \prod_{i=1}^{r} \frac{\gamma_{i}}{\gamma_{i}+1}\right]\right. \\
& +\lambda\left(1-2 F_{Y}\left(y_{2}\right)\right)\left[1-2 \prod_{i=1}^{s} \frac{\gamma_{i}}{\gamma_{i}+1}\right]+\lambda^{2}\left(1-2 F_{Y}\left(y_{1}\right)\right)\left(1-2 F_{Y}\left(y_{2}\right)\right) \\
& \times\left[4\left\{\frac{\gamma_{1} \gamma_{2} \ldots \gamma_{r} \gamma_{r+1} \ldots \gamma_{s}}{\left(\gamma_{1}+2\right)\left(\gamma_{2}+2\right) \ldots\left(\gamma_{r}+2\right)\left(\gamma_{r+1}+1\right) \ldots\left(\gamma_{s}+1\right)}\right\}\right. \\
& \left.\left.-2 \prod_{i=1}^{s} \frac{\gamma_{i}}{\gamma_{i}+1}-2 \prod_{i=1}^{r} \frac{\gamma_{i}}{\gamma_{i}+1}\right]\right] .
\end{aligned}
$$

Therefore, the joint pdf of these concomitants for MTBGED is

$$
\begin{aligned}
f_{[r, s, n, m, k]}(y)= & {\left[\alpha_{2} \theta_{2}\right]^{2} e^{-\theta_{2}\left(y_{1}+y_{2}\right)}\left[\left(1-e^{-\theta_{2} y_{1}}\right)\left(1-e^{-\theta_{2} y_{2}}\right)\right]^{\alpha_{2}-1}\left\{1+\lambda\left(1-2\left(1-e^{-\theta_{2} y_{1}}\right)^{\alpha_{2}}\right)\right.} \\
& \times\left[1-2 \prod_{i=1}^{r} \frac{\gamma_{i}}{\gamma_{i}+1}\right]+\lambda\left(1-2\left(1-e^{-\theta_{2} y_{2}}\right)^{\alpha_{2}}\right) \times\left[1-2 \prod_{i=1}^{s} \frac{\gamma_{i}}{\gamma_{i}+1}\right] \\
& +\lambda^{2}\left(1-2\left(1-e^{-\theta_{2} y_{1}}\right)^{\alpha_{2}}\right)\left(1-2\left(1-e^{-\theta_{2} y_{2}}\right)^{\alpha_{2}}\right) \\
& \times\left[4\left(\frac{\gamma_{1} \gamma_{2} \ldots \gamma_{r} \gamma_{r+1} \ldots \gamma_{s}}{\left(\gamma_{1}+2\right)\left(\gamma_{2}+2\right) \ldots\left(\gamma_{r}+2\right)\left(\gamma_{r+1}+1\right) \ldots\left(\gamma_{s}+1\right)}\right)\right. \\
& \left.\left.-2 \prod_{i=1}^{s} \frac{\gamma_{i}}{\gamma_{i}+1}-2 \prod_{i=1}^{r} \frac{\gamma_{i}}{\gamma_{i}+1}\right]\right\} .
\end{aligned}
$$

Also, the joint mgf of $Y_{[r, n, m, k]}$ and $Y_{[s, n, m, k]}$ is obtained as

$$
\begin{aligned}
M_{[r, s, n, m, k]}\left(t_{1}, t_{2}\right)= & M_{Y}\left(t_{1}\right) M_{Y}\left(t_{2}\right)+\lambda\left[1-2 \prod_{i=1}^{r} \frac{\gamma_{i}}{\gamma_{i}+1}\right]\left[M_{Y}\left(t_{1}\right) M_{Y}\left(t_{2}\right)-M_{Y}\left(t_{1}\right) M_{V}\left(t_{2}\right)\right] \\
+ & \lambda\left[1-2 \prod_{i=1}^{s} \frac{\gamma_{i}}{\gamma_{i}+1}\right]\left[M_{Y}\left(t_{1}\right) M_{Y}\left(t_{2}\right)-M_{V}\left(t_{1}\right) M_{Y}\left(t_{2}\right)\right] \\
+ & \lambda^{2}\left[M_{Y}\left(t_{1}\right)-M_{V}\left(t_{1}\right)\right]\left[M_{Y}\left(t_{2}\right)-M_{V}\left(t_{2}\right)\right] \\
& \times\left[4\left(\frac{\gamma_{1} \gamma_{2} \ldots \gamma_{r} \gamma_{r+1} \ldots \gamma_{s}}{\left(\gamma_{1}+2\right)\left(\gamma_{2}+2\right) \ldots\left(\gamma_{r}+2\right)\left(\gamma_{r+1}+1\right) \ldots\left(\gamma_{s}+1\right)}\right)\right. \\
& \left.-2 \prod_{i=1}^{s} \frac{\gamma_{i}}{\gamma_{i}+1}-2 \prod_{i=1}^{r} \frac{\gamma_{i}}{\gamma_{i}+1}\right] .
\end{aligned}
$$

Differentiating (4.3) with respect $t_{1}$ and $t_{2}$, and putting $t_{1}=t_{2}=0$, we can obtain the product moments $E\left\{Y_{[r, n, m, k]}^{l_{1}} Y_{[s, n, m, k]}^{l_{2}}\right\}=\mu_{[r, s, n, m, k]}^{\left(l_{1}, l_{2}\right)}, l_{1}, l_{2}>0$ as

$$
\begin{aligned}
\mu_{[r, s, n, m, k]}^{\left(l_{1}, l_{2}\right)}= & \mu_{1: 1}^{l_{1}} \mu_{1: 1}^{l_{2}}+\lambda\left[1-2 \prod_{i=1}^{r} \frac{\gamma_{i}}{\gamma_{i}+1}\right]\left[\mu_{1: 1}^{l_{1}} \mu_{1: 1}^{l_{2}}-\mu_{1: 1}^{l_{1}} \mu_{2: 2}^{l_{2}}\right] \\
& +\lambda\left[1-2 \prod_{i=1}^{s} \frac{\gamma_{i}}{\gamma_{i}+1}\right]\left[\mu_{1: 1}^{l_{1}} \mu_{1: 1}^{l_{2}}-\mu_{2: 2}^{l_{1}} \mu_{1: 1}^{l_{2}}\right] \\
& +\lambda^{2}\left[\mu_{1: 1}^{l_{1}}-\mu_{2: 2}^{l_{1}}\right]\left[\mu_{1: 1}^{l_{2}}-\mu_{2: 2}^{l_{2}}\right]
\end{aligned}
$$


Tahmasebi, Jafari, Afshari

$$
\begin{aligned}
& \times\left[4\left(\frac{\gamma_{1} \gamma_{2} \ldots \gamma_{r} \gamma_{r+1} \ldots \gamma_{s}}{\left(\gamma_{1}+2\right)\left(\gamma_{2}+2\right) \ldots\left(\gamma_{r}+2\right)\left(\gamma_{r+1}+1\right) \ldots\left(\gamma_{s}+1\right)}\right)\right. \\
& \left.-2 \prod_{i=1}^{s} \frac{\gamma_{i}}{\gamma_{i}+1}-2 \prod_{i=1}^{r} \frac{\gamma_{i}}{\gamma_{i}+1}\right] .
\end{aligned}
$$

The joint mgf of the concomitants of the $r$-th and $s$-th order statistics, $Y_{[r: n]}$ and $Y_{[s: n]}$, can be deduced from (4.4) with $m=0$ and $k=1$ as

$$
\begin{aligned}
M_{Y_{[r: n]}, Y_{[s: n]}\left(t_{1}, t_{2}\right)=} & \left(\tau_{r}+\tau_{r, s}\right) \alpha_{2}^{2} \operatorname{Beta}\left(\alpha_{2}, 1-\frac{t_{2}}{\theta_{2}}\right)\left[\operatorname{Beta}\left(\alpha_{2}, 1-\frac{t_{1}}{\theta_{2}}\right)-2 \operatorname{Beta}\left(2 \alpha_{2}, 1-\frac{t_{1}}{\theta_{2}}\right)\right] \\
& +\left(1+\tau_{r, s}\right) \alpha_{2}^{2} \operatorname{Beta}\left(\alpha_{2}, 1-\frac{t_{1}}{\theta_{2}}\right) \operatorname{Beta}\left(\alpha_{2}, 1-\frac{t_{2}}{\theta_{2}}\right) \\
& -2\left(\tau_{s}+\tau_{r, s}\right) \alpha_{2}^{2} \operatorname{Beta}\left(\alpha_{2}, 1-\frac{t_{1}}{\theta_{2}}\right) \operatorname{Beta}\left(2 \alpha_{2}, 1-\frac{t_{2}}{\theta_{2}}\right) \\
& +4 \tau_{r, s} \alpha_{2}^{2} \operatorname{Beta}\left(2 \alpha_{2}, 1-\frac{t_{1}}{\theta_{2}}\right) \operatorname{Beta}\left(2 \alpha_{2}, 1-\frac{t_{2}}{\theta_{2}}\right)
\end{aligned}
$$

where $\tau_{r}=\frac{\lambda(n-2 r+1)}{n+1}$ and $\tau_{r, s}=\lambda^{2}\left[\frac{n-2 s+1}{n+1}-\frac{2 r(n-2 s)}{(n+1)(n+2)}\right]$. The product moment $E\left[Y_{[r: n]} Y_{[s: n]}\right]=\mu_{[r, s: n]}$ is obtained (similar to [20]) as

$$
\mu_{[r, s: n]}=\frac{1}{\theta_{2}^{2}}\left\{\left(1+\tau_{r}+\tau_{s}+\tau_{r, s}\right) B^{2}\left(\alpha_{2}\right)-\left(\tau_{r}+\tau_{s}+2 \tau_{r, s}\right) B\left(\alpha_{2}\right) B\left(2 \alpha_{2}\right)+\tau_{r, s} B^{2}\left(2 \alpha_{2}\right)\right\} .
$$

Therefore, the covariance between $Y_{[r: n]}$ and $Y_{[s: n]}$ is given by

$$
\operatorname{Cov}\left(Y_{[r: n]}, Y_{[s: n]}\right)=\frac{D^{2}\left(\alpha_{2}\right)\left[\tau_{r, s}-\tau_{r} \tau_{s}\right]}{\theta_{2}^{2}}
$$

Thus the $r$-th and $s$-th concomitants are positively correlated and its value decreases as $r$ and $s$ pull apart. Finally, the coefficient of correlation between $Y_{[r: n]}$ and $Y_{[s: n]}$ is derived as

$$
\rho_{[r, s: n]}=\frac{D^{2}\left(\alpha_{2}\right)\left[\tau_{r, s}-\tau_{r} \tau_{s}\right]}{\left\{\left[C\left(\alpha_{2}\right)+\tau_{r}\left(C\left(2 \alpha_{2}\right)-C\left(\alpha_{2}\right)\right)\right]\left[C\left(\alpha_{2}\right)+\tau_{s}\left(C\left(2 \alpha_{2}\right)-C\left(\alpha_{2}\right)\right)\right]\right\}^{\frac{1}{2}}} .
$$

The values of $\rho_{[r, s: n]}$ for $n=10$ and some values of $\alpha_{2}$ and $\lambda$ are given in Table 1 . We can conclude that $\rho_{[r, s: n]}$ has minimum value when $r=1$ and $s=10$, and it has maximum value when $r=5$ and $s=6$ for given $\alpha_{2}$ and $\lambda$.

Remark 4.1. Set $m=-1$ in (4.3) and (4.4), we can obtain the joint mgf and product moments of two concomitants of $k$-th lower record values of MTBGED.

\section{References}

[1] M. Ahsanullah, A characterization of the uniform distribution by dual generalized order statistics, Communications in Statistics - Theory and Methods 33(12) (2004) 2921-2928.

[2] M. Ahsanullah, On lower generalized order statistics and a characterization of power function distribution, Statistical Methods 7(1) (2005) 16-28.

[3] M. Ahsanullah and M. I. Beg, Concomitant of generalized order statistics in gumbel's bivariate exponential distribution, Journal of Statistical Theory and Applications 6(2) (2006) 118-132.

[4] G. Arslan, On a characterization of the uniform distribution by generalized order statistics, Journal of Computational and Applied Mathematics 235(16) (2011) 4532-4536. 
[5] H. Athar, R. Khan and Z. Anwar, Exact moments of lower generalized order statistics from power function distribution, Bulletin of the Calcutta Statistical Association 62(245) (2010) 245-246.

[6] K. Balasubramanian and M. I. Beg, Concomitants of order statistics in Morgenstern type bivariate exponential distribution, Journal of Applied Statistical Science 54(4) (1997) 233-245.

[7] H. M. Barakat and M. E. El-Adll, Asymptotic theory of extreme dual generalized order statistics, Statistics \& Probability Letters 79(9) (2009) 1252-1259.

[8] M. I. Beg and M. Ahsanullah, Concomitants of generalized order statistics from Farlie-GumbelMorgenstern distributions, Statistical Methodology 5(1) (2008) 1-20.

[9] M. Burkschat, E. Cramer and U. Kamps, Dual generalized order statistics, Metron LXI(1) (2003) 1326.

[10] E. J. Gumbel, Bivariate exponential distributions, Journal of the American Statistical Association 55(292) (1960) 698-707.

[11] R. D. Gupta and D. Kundu, Generalized exponential distributions, Australian \& New Zealand Journal of Statistics 41(2) (1999) 173-188.

[12] U. Kamps, A concept of generalized order statistics (B.G. Teubner Stuttgart, Germany, 1995).

[13] A. Khan, Z. Anwar and S. Chishti, Characterization of continuous distributions through conditional expectation of functions of dual generalized order statistics, Pakistan Journal of Statistics 26(4) (2010).

[14] R. Khan and D. Kumar, On moments of lower generalized order statistics from exponentiated Pareto distribution and its characterization, Applied Mathematical Sciences (Ruse) 4(55) (2010) 2711-2722.

[15] R. Khan and D. Kumar, Expectation identities of lower generalized order statistics from generalized exponential distribution and a characterization, Mathematical Methods of Statistics 20(2) (2011) 150157.

[16] A. Mbah and M. Ahsanullah, Some characterizations of the power function distribution based on lower generalized order statistics, Pakistan Journal of Statistics 23(2) (2007) 139-146.

[17] D. Morgenstern, Einfache beispiele zweidimensionaler verteilungen, Mitteilingsblatt für Mathematishe Statistik 8(1) (1956) 234-235.

[18] Nayabuddin, Concomitants of dual generalized order statistics from Farlie Gumbel Morgenstern type bivariate power function distribution, Journal of Global Research in Mathematical Archives 1(8) (2013) 79-90.

[19] P. Pawlas and D. Szynal, Recurrence relations for single and product moments of lower generalized order statistics from the inverse Weibull distribution, Demonstratio Mathematica XXXIV(2) (2001) 353-358.

[20] S. Tahmasebi and A. A. Jafari, Concomitants of order statistics and record values from Morgenstern type bivariate generalized exponential distribution, Bulletin of the Malaysian Mathematical Sciences Society (Accepted for publication) (2013). 
Table 1. The values of $\rho_{[r, s: n]}$ for $n=10$ and some values of $\alpha_{2}$ and $\lambda$.

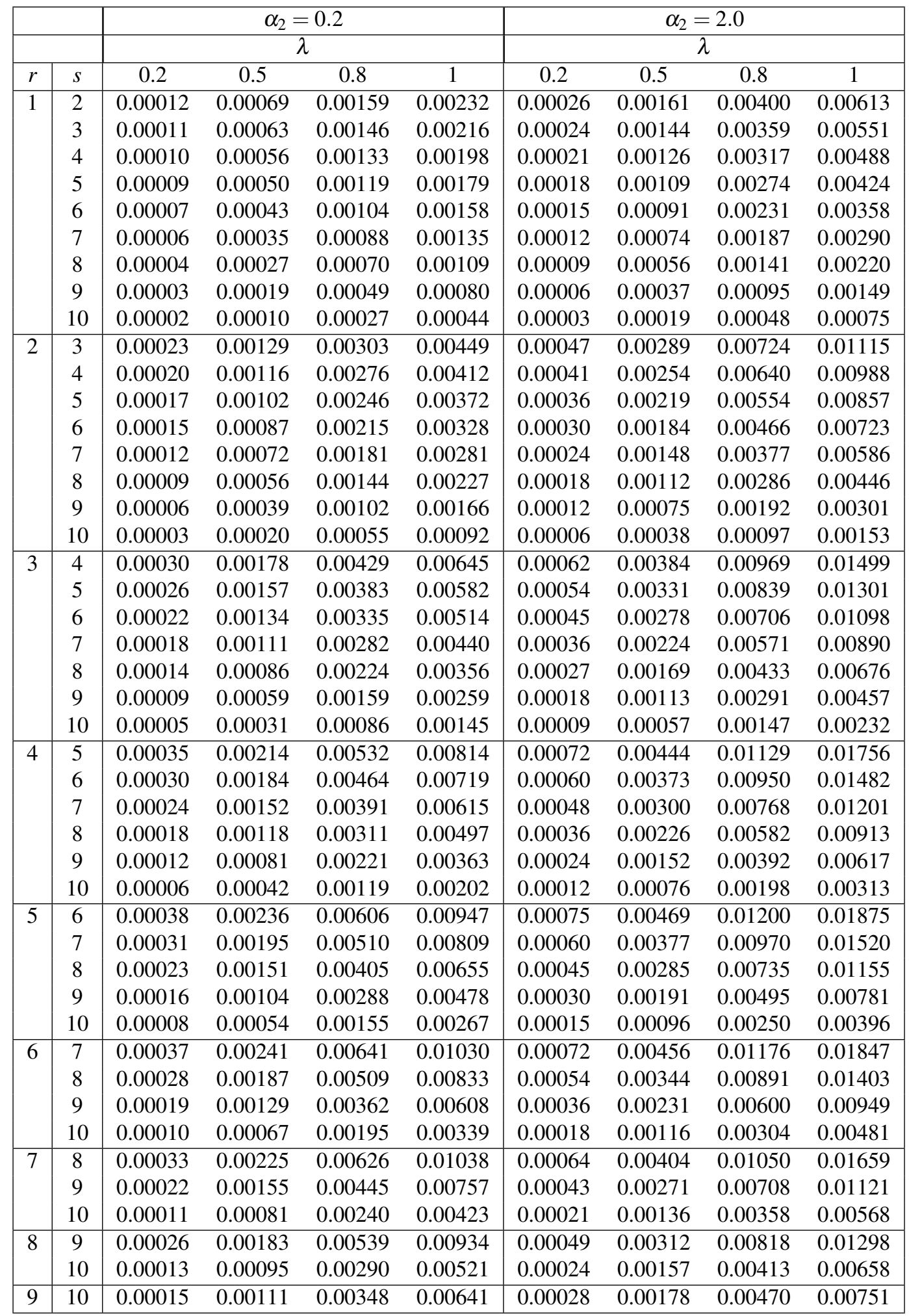

